\title{
Synergic effects of histology subtype, tumor size, and lymph node metastasis on distant metastasis in differentiated thyroid cancer
}

\author{
Zeming Liu ${ }^{1 \#}$, Sichao Chen ${ }^{1 \#}$, Yihui Huang ${ }^{1}, \mathrm{Di}_{\mathrm{Hu}}{ }^{1}$, Wen Zeng ${ }^{2}$, Min Wang $^{1}$, Wei Zhou ${ }^{1}$, \\ Danyang Chen ${ }^{1}$, Haifeng Feng ${ }^{1}$, Wei Wei ${ }^{3}$, Chao Zhang ${ }^{4}$, Ling Zhou ${ }^{1 \#}$, Liang Guo ${ }^{1 \#}$ \\ ${ }^{1}$ Department of Plastic Surgery, ${ }^{2}$ Department of Ophthalmology, Zhongnan Hospital of Wuhan University, Wuhan 430071, China; ${ }^{3}$ Department \\ of Pediatrics, St John Hospital and Medical Center, Detroit, MI, USA; ${ }^{4}$ Department of Cardiovascular Surgery, Union Hospital, Tongji Medical \\ College, Huazhong University of Science and Technology, Wuhan 430033, China \\ Contributions: (I) Conception and design: All authors; (II) Administrative support: M Wang, W Zhou, D Chen; (III) Provision of study materials \\ or patients: Z Liu; (IV) Collection and assembly of data: H Feng, W Wei, C Zhang; (V) Data analysis and interpretation: S Chen, Y Huang; (VI) \\ Manuscript writing: All authors; (VII) Final approval of manuscript: All authors. \\ \#These authors contributed equally to this work. \\ Correspondence to: Liang Guo; Ling Zhou. Department of Plastic Surgery, Zhongnan Hospital of Wuhan University, Donghu Road 169, Wuchang \\ District, Wuhan 430071, China. Email: guoliangwhzn@163.com; zhoulingwhzn@163.com.
}

Background: Detection of distant metastasis (DM) is important in differentiated thyroid cancer (DTC). This study aimed to investigate the synergic effects of histology subtype, tumor size, and lymph node metastasis (LNM) status on the occurrence of DM in DTC.

Methods: We collected data of 96,788 patients with DTC. Univariate and multivariate analyses were conducted to identify the risk factors of DM. Relative excess risk of synergic effect, attributable proportion of synergic effect, and synergy index were then calculated to assess synergic effects. Further, Kaplan-Meier method using the log-rank test and receiver operating characteristic (ROC) curves were utilized.

Results: Age at diagnosis $(\mathrm{P}<0.001)$, sex $(\mathrm{P}<0.001)$, race $(\mathrm{P}<0.01)$, tumor size $(\mathrm{P}<0.001)$, N stage $(\mathrm{P}<0.001)$, histology subtype $(\mathrm{P}<0.001)$, and extrathyroidal extension $(\mathrm{P}<0.001)$ were risk factors for $\mathrm{DM}$ in both univariate and multivariate analyses. We also found a significant additive synergic effect between histology subtype and LNM, and between tumor size and LNM on DM in the DTC patients. In addition, patients with follicular thyroid cancer and N1 stage had the sharpest decline in cancer-specific survival curves $(\mathrm{P}<0.001)$ and all-cause survival curves $(\mathrm{P}<0.001)$ compared to patients with other combinations of histology subtype and $\mathrm{N}$ stage. Similar results were obtained in patients with larger tumors $(\geq 10 \mathrm{~mm})$ and $\mathrm{N} 1 \mathrm{stage}$. The areas under the curve of histology subtype, tumor size, and LNM status were $0.569,0.744$, and 0.681 , respectively.

Conclusions: Age at diagnosis, sex, race, tumor size, N stage, histology subtype, and extrathyroidal extension are risk factors for DM in DTC patients. LNM has a synergic effect with either follicular thyroid histology or larger tumor size for higher risk of DM which is important for diagnosing DM.

Keywords: differentiated thyroid cancer (DTC); distant metastasis (DM); synergic effect; Surveillance, Epidemiology, and End Results (SEER)

Submitted Jun 13, 2019. Accepted for publication Sep 11, 2019.

doi: $10.21037 /$ atm.2019.09.137

View this article at: http://dx.doi.org/10.21037/atm.2019.09.137 


\section{Introduction}

The incidence of thyroid cancer has continuously increased in recent years (1-4) primarily due to the widespread use of diagnostic imaging, particularly ultrasound. Differentiated thyroid cancer (DTC) (5), which consists of papillary thyroid cancer (PTC) and follicular thyroid cancer (FTC), accounts for more than $85 \%$ of thyroid cancers. DTC has relatively good prognosis, with 10 -year survival rates of approximately $90-95 \%$ (6). However, approximately $1.2-4.2 \%$ of DTC patients develop distant metastasis (DM) (7-9), which is the most frequent cause of thyroid cancerrelated death. Consequently, early detection of DM is important. DM in DTC usually occurs in the lung and/or bone, but some DTC patients have DM of the liver, brain, and skin (10). In general, FTC patients are at higher risk of DM, while PTC patients have a higher risk of lymph node metastasis (LNM) $(11,12)$. Some studies have also identified tumor size and LNM as risk factors for DM in DTC patients $(13,14)$. However, these studies only evaluated the relationship between these factors and DM in DTC. Studies aiming to investigate the synergic effect of these factors on DM are rare. Therefore, this study aimed is to investigate the synergic effect of histology subtype, tumor size, and LNM on DM in DTC patients.

\section{Methods}

\section{Data collection}

This retrospective study was approved by the Ethics Committee of Zhongnan Hospital of Wuhan University and complied with the ethical standards of the Declaration of Helsinki as well as the relevant national and international guidelines, and informed consent was obtained from all the patients. We recruited patients with DTC from the Surveillance, Epidemiology, and End Results (SEER) database of the National Cancer Institute. This is an openaccess database that contains data about the demographic, pathological, primary tumor, and treatment characteristics of cancer patients. Patients with incomplete DM data were excluded. In total, 96,788 DTC patients diagnosed between 2004 and 2013, all of whom were followed-up until December 2013, were included in the study.

Demographic characteristics consisted of sex (male or female), race (white, black, other), and age at diagnosis $(<55$ or $\geq 55$ years). Pathological characteristics included tumor size ( $<10$ or $\geq 10 \mathrm{~mm}$ ), LNM (N0 or N1 stage), DM (M0 or M1 stage), multifocality, histology subtype (PTC or FTC), and extrathyroidal extension. Treatment characteristics included radiation therapy (none or refused, radiation beam or radioactive implants, and radioisotopes as well as radiation beam plus isotopes or implants), and surgery (none, lobectomy, subtotal or nearly total thyroidectomy, and total thyroidectomy).

\section{Statistical analysis}

All included patients were divided into the DM and the non-DM groups. To explore factors associated with DM, clinicopathological characteristics were compared using the Chi-squared test for univariate analysis and binary logistic regression for multivariate analysis.

Patients were then divided into different groups based on two factors at a time: histology subtype and $\mathrm{N}$ stage; tumor size and $\mathrm{N}$ stage; and histology subtype and tumor size. To assess the synergic effect of the two factors on DM occurrence, logistic regression modeling and $95 \%$ confidence intervals (CIs) of the odds ratios (ORs) were calculated, with adjustment for other cofounding variables. The relative excess risk (RERI), attributable proportion (AP), and synergy index (SI) were calculated to evaluate the synergic effect of these factors on DM in DTC patients. A $95 \%$ CI of 0 and SI of 1 for RERI and AP indicated no additive synergic effect. That is, RERI $>0, \mathrm{AP}>0$, or $\mathrm{S}>1$ is considered to verify the existence of a synergic effect. The Kaplan-Meier method using the log-rank test was utilized to evaluate the influence of clinicopathological factors on patients' survival, and receiver operating characteristic (ROC) curves were plotted to analyze the predictive value of each variable on DM.

All $\mathrm{P}$ values were two-sided, with $\mathrm{P}<0.05$ being considered statistically significant. Statistical analyses were performed using SPSS version 24.0 (IBM Corp., Armonk, NY, USA), R statistical software (R Core Development Team, Vienna, Austria), and GraphPad Prism version 6 (GraphPad Software Inc., La Jolla, CA, USA).

\section{Results}

\section{General characteristics of the study population}

The general clinicopathological characteristics of the 96,788 patients analyzed in this study are shown in Table 1. FTC patients accounted for only $5.9 \%$ of this population. The mean age at diagnosis was 49 years. There were $31,525(34.3 \%)$ patients with tumors smaller than $10 \mathrm{~mm}, 20,963(21.9 \%)$ 
Table 1 Demographic and clinicopathologic characteristics of 96,788 patients with DTC

\begin{tabular}{|c|c|}
\hline Characteristics & Number (\%) \\
\hline \multicolumn{2}{|l|}{ Age at diagnosis (years) } \\
\hline Mean [range] & 49 [2-105] \\
\hline$<55$ & $60,841(62.9)$ \\
\hline$\geq 55$ & $35,947(37.1)$ \\
\hline \multicolumn{2}{|l|}{ Sex } \\
\hline Female & $74,325(76.8)$ \\
\hline Male & $22,463(23.2)$ \\
\hline \multicolumn{2}{|l|}{ Race } \\
\hline White & $79,063(82.7)$ \\
\hline Black & $6,299(6.6)$ \\
\hline Other & $10,246(10.7)$ \\
\hline \multicolumn{2}{|l|}{ Tumor size (mm) } \\
\hline Mean [range] & 18.59 [0-988] \\
\hline$<10$ & 31,525 (34.3) \\
\hline$\geq 10$ & $60,451(65.7)$ \\
\hline \multicolumn{2}{|l|}{$\mathrm{N}$ stage } \\
\hline No & $74,693(78.1)$ \\
\hline $\mathrm{N} 1$ & $20,963(21.9)$ \\
\hline Distant metastasis & $1,488(1.5)$ \\
\hline Multifocality & $37,584(39.9)$ \\
\hline \multicolumn{2}{|l|}{ Histology subtype } \\
\hline PTC & $91,070(94.1)$ \\
\hline FTC & $5,718(5.9)$ \\
\hline Extrathyroidal extension & $15,339(16.0)$ \\
\hline \multicolumn{2}{|l|}{ Radiation therapy } \\
\hline None or refused & $48,030(50.8)$ \\
\hline Radiation beam or radioactive implants & $1,831(1.9)$ \\
\hline $\begin{array}{l}\text { Radioisotopes or radiation beam plus } \\
\text { isotopes or implants }\end{array}$ & $44,761(47.3)$ \\
\hline \multicolumn{2}{|l|}{ Surgery } \\
\hline Lobectomy & $13,723(14.7)$ \\
\hline Subtotal or near-total thyroidectomy & $3,572(3.8)$ \\
\hline Total thyroidectomy & $76,075(81.5)$ \\
\hline
\end{tabular}

DTC, differentiated thyroid cancer; PTC, papillary thyroid cancer; FTC, follicular thyroid cancer. patients with LNM, and 1,488 (1.5\%) patients with DM.

\section{Factors associated with DM in DTC}

Univariate analysis showed that larger tumors $(\mathrm{P}<0.001)$ and LNM $(\mathrm{P}<0.001)$ were associated with a higher risk of DM (Table 2). In addition, FTC patients may have a higher risk of DM than PTC patients, $(\mathrm{P}<0.001)$. Moreover, age at diagnosis, sex, race, multifocality, and extrathyroidal extension were significantly associated with DM in DTC patients (all $\mathrm{P}<0.001$ ).

In the multivariate analysis, age at diagnosis, sex, race, tumor size, $\mathrm{N}$ stage, histology subtype, and extrathyroidal extension were found to be independent risk factors for DM. It was noticeable that FTC (OR: 6.266, 95\% CI: $5.176-7.586, \mathrm{P}<0.001)$, larger tumor size $[>10 \mathrm{~mm}(\mathrm{OR}$ : 2.433, 95\% CI: $1.910-3.098, \mathrm{P}<0.001]$, and $\mathrm{N} 1$ stage (OR: 3.998, 95\% CI: $3.415-4.681, \mathrm{P}<0.001)$ increased the risks of DM (Table 3).

\section{Synergic effect of histology subtype, tumor size, and LNM on DM}

To comprehensively explore the synergic effects of histology subtype, tumor size, and LNM status on DM, the patients were divided into 4 groups according to two factors at a time (histology subtype and tumor size; histology subtype and $\mathrm{N}$ stage; and tumor size and $\mathrm{N}$ stage). In the histology and subtype combination group, patients with FTC and N1 stage (OR: 33.495, 95\% CI: 22.402-50.083, $\mathrm{P}<0.001$ ) had the highest risk of DM (Table 4), after adjusting for age at diagnosis, sex, race, tumor size, and extrathyroidal extension. In addition, the risk of DM was higher in patients with FTC and N0 stage (OR: 5.685, 95\% CI: 4.576-7.062, $\mathrm{P}<0.001$ ), or patients with PTC and N1 stage (OR: 3.801, 95\% CI: 3.224-4.482, P<0.010) than in patients with PTC and N0 stage after the adjustment.

Based on the abovementioned results, the RERI was 76.973 (95\% CI: 50.238-103.708), which indicates that there would be 76.973 relative excess risks contributed by the additive synergic effect of FTC histology subtype and LNM status on the presence of DM. The AP was 0.864 (95\% CI: $0.823-0.905$ ), which suggested that $86.4 \%$ of DM exposed to the two risk factors was caused by the synergic effect. In addition, the value of SI was larger than 1 (7.930, 95\% CI: 5.833-10.780), which signified the existence of a 
Table 2 The relationship between clinicopathological factors and distant metastasis in DTC

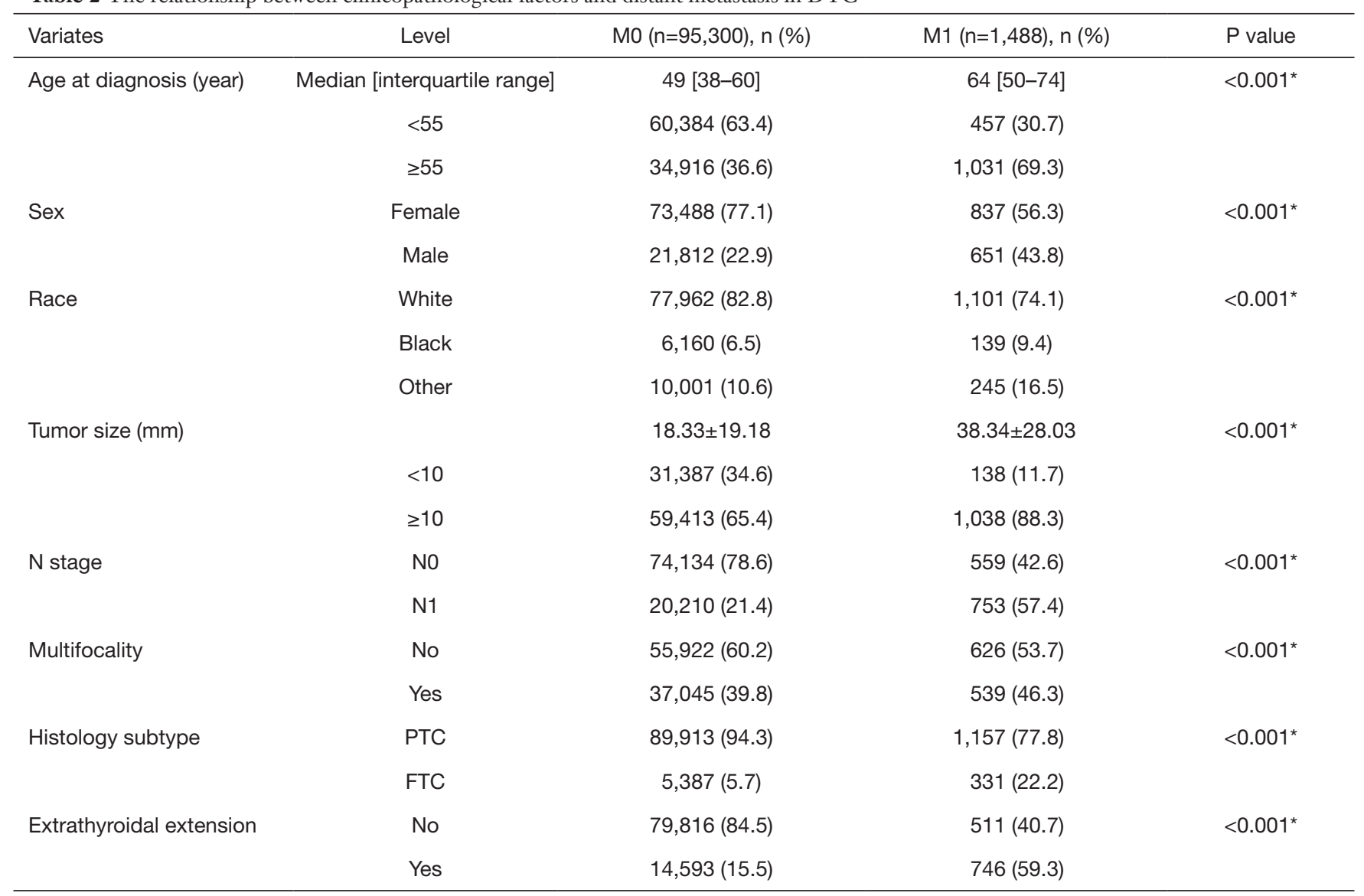

*, P value <0.05. DTC, differentiated thyroid cancer; PTC, papillary thyroid cancer; FTC, follicular thyroid cancer.

synergic effect between FTC histology subtype and LNM status on the presence of DM.

After adjustment for age, sex, race, histology subtype, and extrathyroidal extension, the risk of DM was the highest in DTC patients with larger tumors $(\geq 10 \mathrm{~mm})$ and N1 stage (OR: 13.584, 95\% CI: 9.617-19.189, $\mathrm{P}<0.001)$ compared with those of other combinations of tumor size and $\mathrm{N}$ stage (Table 5). The risk of DM was also higher in patients with only larger tumors (OR: 4.979, 95\% CI: 3.553-6.978, $\mathrm{P}<0.001$ ) or LNM (OR: 7.807, 95\% CI: 4.978-12.242, $\mathrm{P}<0.001)$ compared to patients without larger tumors and LNM. Moreover, there would be 5.058 relative excess risks contributed by the additive synergic effect of large tumor size and LNM on the presence of DM. In addition, exposure to the two risk factors created an additive synergic effect and caused $35.7 \%$ of DM. Then, 20 and $40 \mathrm{~mm}$ were also set as the cut-off value to explore the synergic effect between tumor size and LNM and it yielded similar results
(Tables $S 1, S 2)$.

We also evaluated the synergic effect between histology subtype and tumor size on the presence of DM (Table S3). However, the RERI ( $-14.686,95 \%$ CI: -24.964 to -4.409$)$ and $\mathrm{AP}(-1.119,95 \% \mathrm{CI}:-1.912$ to -0.327$)$ were lower than 0 , indicating no additive synergic effect between these two factors.

\section{Synergic effect of histology subtype, tumor size, and LNM on survival in DTC}

According to the Kaplan-Meier analysis of the four groups divided by histology subtype and LNM status (Figure 1A), the cancer-specific survival curve of patients with PTC and N0 stage was relatively flat, while that of patients with FTC or N1 stage showed a modest decline in the survival curve. By contrast, patients with FTC and N1 stage showed a sharp decline in cancer-specific survival. Similar results 
Table 3 Multivariate analysis of distant metastases in DTC

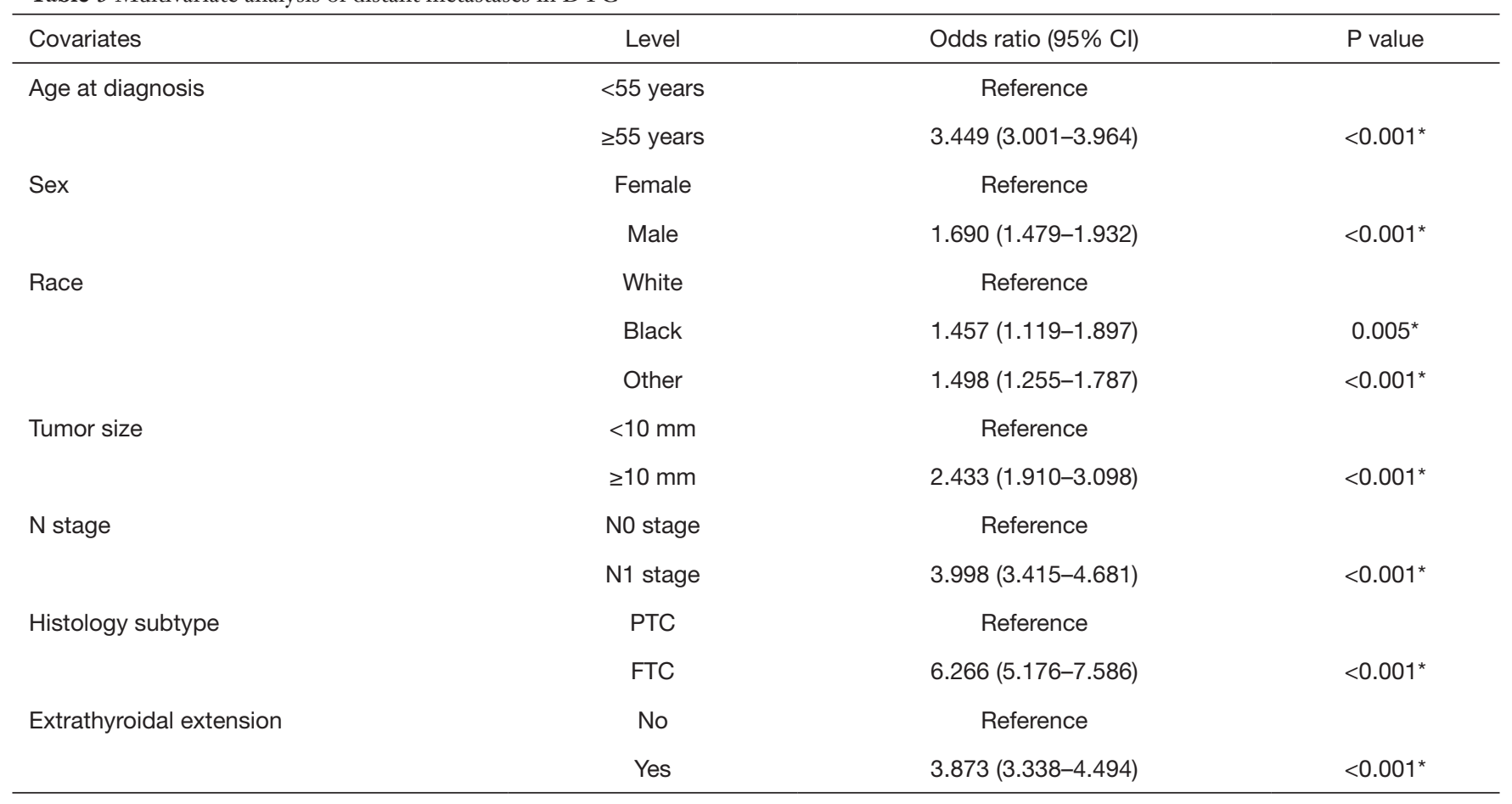

*, P value <0.05. DTC, differentiated thyroid cancer; PTC, papillary thyroid cancer; FTC, follicular thyroid cancer.

Table 4 Measures for estimation of synergic effect between histology subtype and N stage for the risk of distant metastasis in DTC

\begin{tabular}{lcccc}
\hline Histology subtype & N stage & M1 case $(\%)$ & Total case & Odds ratio $(95 \% \mathrm{Cl})$ \\
\hline PTC & 0 & $371(0.5)$ & 69,343 & Reference \\
& 1 & $684(3.3)$ & 20,750 & $3.801(3.224-4.482)$ \\
FTC & 0 & $188(3.5)$ & 5,350 & $5.685(4.576-7.062)$ \\
& 1 & $69(32.4)$ & 213 & $33.495(22.402-50.083)$ \\
RERI & & & $76.973(50.238-103.708)$ & $<0.001^{*}$ \\
AP & & $0.864(0.823-0.905)$ & & $<0.001^{*}$ \\
SI & & $7.930(5.833-10.780)$ & & \\
\hline
\end{tabular}

Adjusted for age at diagnosis, sex, race, tumor size, extrathyroidal extension. *, represent the $\mathrm{P}$ value $<0.05$. DTC, differentiated thyroid cancer; PTC, papillary thyroid cancer; CI, confidence interval; FTC, follicular thyroid cancer; RERI, relative excess risk; AP, attributable proportion; SI, synergy index.

were obtained in analysis of all-cause survival (Figure 1B).

Similarly, based on the Kaplan-Meier analysis of the four groups divided by tumor size and LNM status, the cancer-specific survival curve and all-cause survival curve of patients with larger tumor size $(\geq 10 \mathrm{~mm})$ and $\mathrm{N} 1$ stage showed a remarkable decline compared to that of patients with larger tumor size or N1 stage and that of DTC patients with smaller tumors and N0 stage (Figure 2A,B).

\section{ROC curve analysis and predictive value assessment}

The ROC curve was plotted to determine the predictive value of histology subtype, tumor size, and LNM status for the presence of DM in DTC patients. As shown in Figure 3, the areas under the curve of histology subtype, tumor size, and LNM status were 0.569, 0.744, and 0.681, respectively, showing their good predictive value. 
Table 5 Measures for estimation of synergic effect between tumor size and N stage for the risk of distant metastasis in DTC

\begin{tabular}{lcccc}
\hline Tumor size & N stage & M1 case $(\%)$ & Total case & Odds ratio $(95 \% \mathrm{Cl})$ \\
\hline$<10 \mathrm{~mm}$ & 0 & $71(0.3)$ & 28,048 & Reference \\
& 1 & $53(1.6)$ & 3,274 & $7.807(4.978-12.242)$ \\
$\geq 10 \mathrm{~mm}$ & 0 & $393(0.9)$ & 43,168 & $4.979(3.553-6.978)$ \\
& 1 & $579(3.5)$ & 16,689 & $13.584(9.617-19.189)$ \\
RERI & & $5.058(2.713-7.403)$ & $<0.001^{*}$ \\
AP & & $0.357(0.219-0.496)$ & \\
SI & & $1.624(1.275-2.069)$ & \\
\hline
\end{tabular}

Adjusted for age at diagnosis, sex, race, histology subtype, extrathyroidal extension. *, represent the $\mathrm{P}$ value $<0.05$. DTC, differentiated thyroid cancer; $\mathrm{Cl}$, confidence interval; RERI, relative excess risk; AP, attributable proportion; SI, synergy index.

A

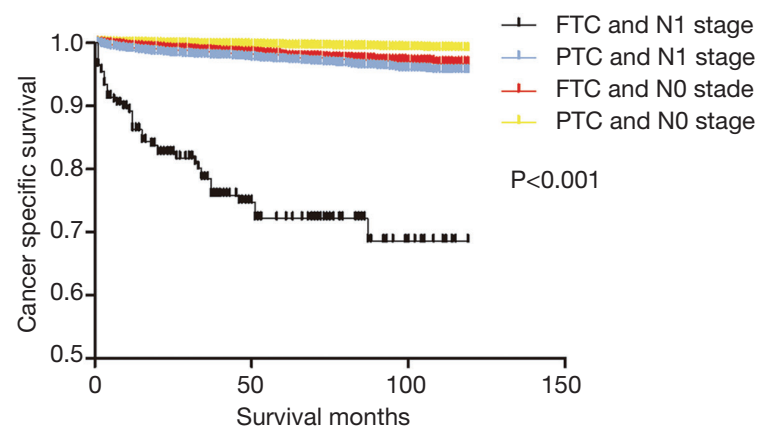

B

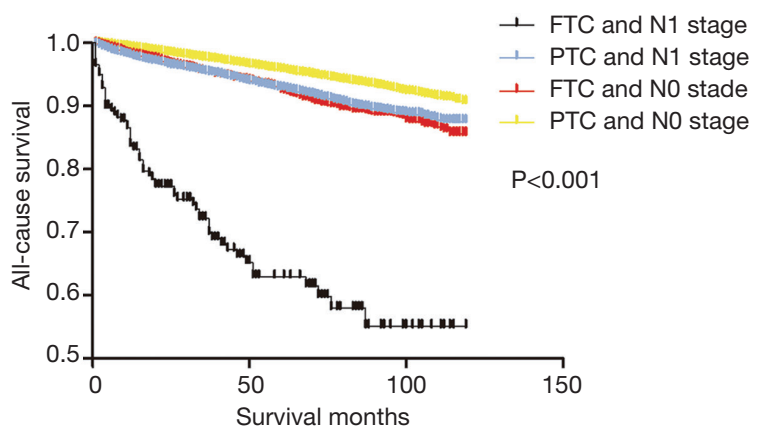

Figure 1 The synergic effects of histology subtype and N stage on the prognosis of DTC patients. (A) Effects of histology subtype and $\mathrm{N}$ stage on cancer-specific survival of patients with DTC. (B) Effects of histology subtype and $\mathrm{N}$ stage on overall survival of patients with DTC. FTC, follicular thyroid cancer; DTC, differentiated thyroid cancer.

A

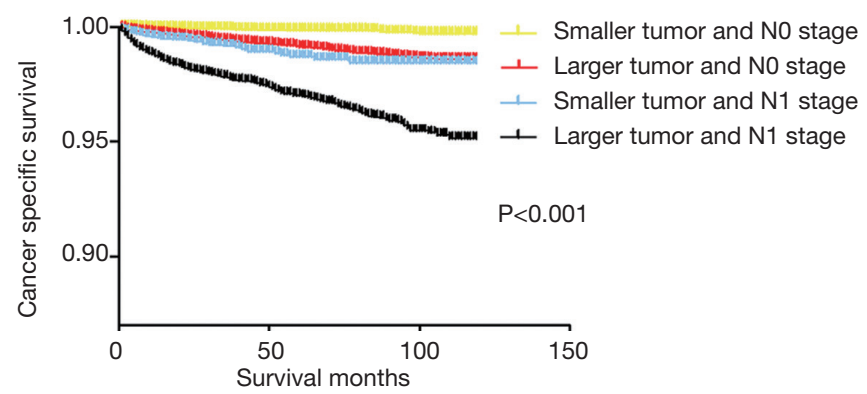

B

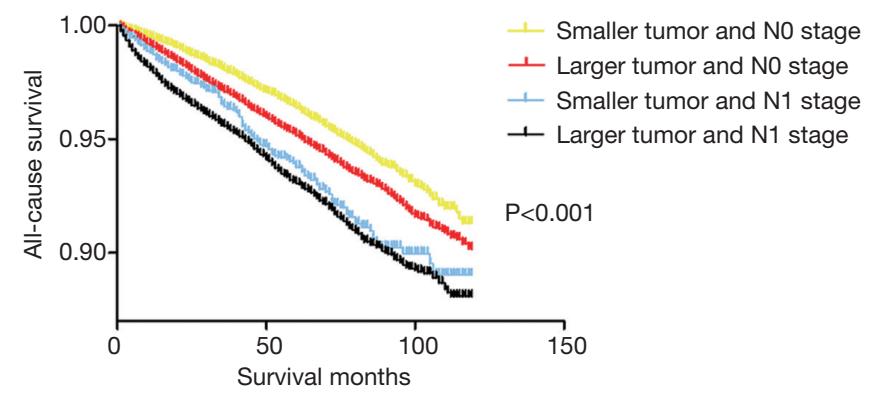

Figure 2 The synergic effects of tumor size and N stage on the prognosis of DTC patients. (A) Effects of tumor size and N stage on cancer specific survival of patients with DTC. (B) Effects of tumor size and N stage on overall survival of patients with DTC. DTC, differentiated thyroid cancer. 


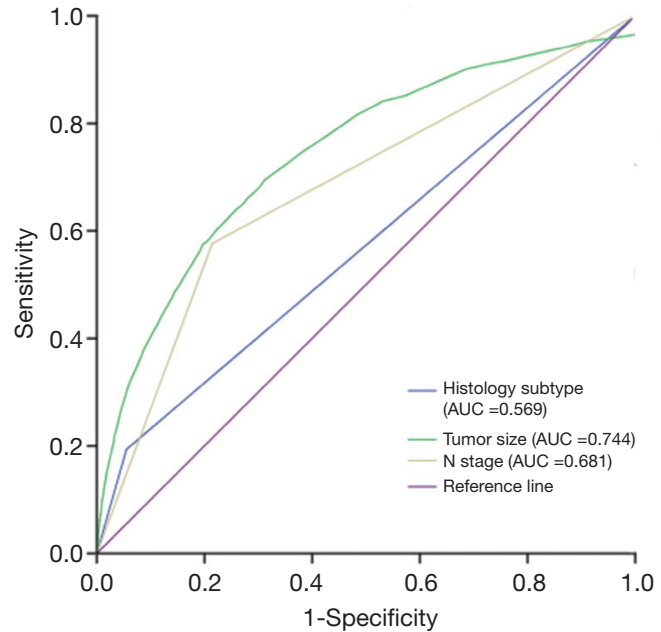

Figure 3 ROC curve for determining the predictive power of histology subtype, tumor size and LNM on DM in DTC. ROC, receiver operating characteristic; AUC, area under curve; LNM, lymph node metastasis; DM, distant metastasis.

\section{Conclusions}

The SEER database, which includes data from approximately $28 \%$ of the US population, is identified as the gold standard database for cancer analysis in this country (15-18). Therefore, the present study was able to enroll a large number of patients to evaluate the influencing factors of DM in DTC and any synergic effects among them.

DM was observed in $1.5 \%$ of DTC patients in our study, which is consistent with previously reported incidences of $1.2-4.2 \%$ in patients with DTC (7-9). Although DM rarely occurs in DTC, DM is the main cancer-specific cause of mortality in thyroid cancer patients and greatly reduces the cancer-specific survival, and this has not been improved in the past 20 years $(19,20)$. In our study, DTC patients with FTC and LNM and patients with larger tumors and LNM had a higher risk of DM and showed a sharper decline in both cancer-specific survival and overall survival, highlighting the importance of early detection of DM.

In this study, both univariate and the multivariate analyses showed that histology subtype of FTC, larger tumor size, and LNM significantly increased the risks of DM in DTC patients. Previous studies also suggested that DM is more frequent in FTC than in PTC $(11,12)$. Franssila reported that this may be because blood vessel invasion is more common in FTC than in PTC. By contrast, islets of PTC often appear in intrathyroid lymphatic vessels (12). Vuong et al. evaluated 73,219 DTC patients and also reported that tumor size and LNM are significant risk factors for DM in (13).

In our study, we also found that there were significant synergic effects between histology subtype and LNM, as well as between tumor size and LNM, on DM in DTC. The incidence of DM in patients with FTC and N1 stage was $32.4 \%$, which is almost 10 times higher than that of patients with FTC and N0 stage or that of patients with PTC and N1 stage (3.5\% and 3.3\%, respectively). Similarly, we also found that a markedly higher incidence of DM in patients with larger tumors and LNM than in patients with only larger tumors or LNM. The synergic effect of these two factors caused $86.4 \%$ of DM in patients with FTC and LNM and $35.7 \%$ of DM in patients with larger tumors and LNM.

However, the precise mechanism to explain the results of this study is yet to be determined, although we hypothesize that the synergic effect between FTC and LNM on DM might be due to the mutual promotion of both blood vessel and lymphatic channel invasion. In addition, large tumor size has been reported to be a risk factor for cervical LNM in DTC $(21,22)$, further supporting the existence of a synergic effect between larger tumor size and LNM.

Treatment strategies differ significantly between DTC patients with and without DM. DTC patents without DM are managed via local therapeutic measures, such as surgery and radiofrequency or cryogenic ablation. Unfortunately, once DM occurs in DTC, either surgical treatment or adjuvant I-131 treatment seem not to play an important role (Table S4). Therefore, timely diagnosis of DM according to its risk factors is crucial for optimal treatment and improving prognosis. Consequently, careful observation and evaluation via imaging assessment should be considered for FTC patients with LNM or DTC patients with larger tumor size and LNM.

The present study has some limitations. First, selection bias could not be ruled out due to the retrospective design. Second, we cannot explain our results in terms of pathological mechanisms with absolute certainty. Moreover, lateral LNM and central LNM were not discussed separately due to insufficiency of the data. Unlike lateral LNM, central LNM has been reported to have no association with the risk DM (13). Further, other meaningful clinicopathologic characteristics, such as number of nodal metastases, node size, presence of 
extranodal extension, were not included in the analysis.

In conclusion, age at diagnosis, sex, race, tumor size, $\mathrm{N}$ stage, histology subtype, and extrathyroidal extension are risk factors for DM in DTC patients. FTC and LNM or larger tumor size and LNM have a synergic effect for higher risk of DM, which is important for early detection of DM.

\section{Acknowledgments}

None.

\section{Footnote}

Conflicts of Interest: The authors have no conflicts of interest to declare.

Ethical Statement: The authors are accountable for all aspects of the work in ensuring that questions related to the accuracy or integrity of any part of the work are appropriately investigated and resolved. This retrospective study was approved by the Ethics Committee of Zhongnan Hospital of Wuhan University (No. 2019010) and complied with the ethical standards of the Declaration of Helsinki as well as the relevant national and international guidelines, and informed consent was obtained from all the patients.

\section{References}

1. Lim H, Devesa SS, Sosa JA, et al. Trends in Thyroid Cancer Incidence and Mortality in the United States, 1974-2013. JAMA 2017;317:1338-48.

2. Davies L, Welch HG. Current thyroid cancer trends in the United States. JAMA Otolaryngol Head Neck Surg 2014;140:317-22.

3. Chen $W$, Zheng R, Baade PD, et al. Cancer statistics in China, 2015. CA Cancer J Clin 2016;66:115-32.

4. Siegel RL, Miller KD, Jemal A. Cancer statistics, 2018. CA Cancer J Clin 2018;68:7-30.

5. Mao Y, Xing M. Recent incidences and differential trends of thyroid cancer in the USA. Endocr Relat Cancer 2016;23:313-22.

6. Tuttle RM, Morris LF, Haugen BR, et al. Perrier 2017 AJCC Cancer Staging Manual. 8th edition. Springer Switzerland, 2017.

7. Lang BH, Wong KP, Cheung CY, et al. Evaluating the prognostic factors associated with cancer-specific survival of differentiated thyroid carcinoma presenting with distant metastasis. Ann Surg Oncol 2013;20:1329-35.

8. Kim HJ, Lee JI, Kim NK, et al. Prognostic implications of radioiodine avidity and serum thyroglobulin in differentiated thyroid carcinoma with distant metastasis. World J Surg 2013;37:2845-52.

9. Kim H, Kim YN, Kim HI, et al. Preoperative serum thyroglobulin predicts initial distant metastasis in patients with differentiated thyroid cancer. Sci Rep 2017;7:16955.

10. Schlumberger M, Challeton C, De Vathaire F, et al. Radioactive iodine treatment and external radiotherapy for lung and bone metastases from thyroid carcinoma. J Nucl Med 1996;37:598-605.

11. Mizukami Y, Michigishi T, Nonomura A, et al. Distant metastases in differentiated thyroid carcinomas: a clinical and pathologic study. Hum Pathol 1990;21:283-90.

12. Franssila KO. Is the differentiation between papillary and follicular thyroid carcinoma valid? Cancer 1973;32:853-64.

13. Vuong HG, Duong UNP, Pham TQ, et al. Clinicopathological Risk Factors for Distant Metastasis in Differentiated Thyroid Carcinoma: A Meta-analysis. World J Surg 2018;42:1005-17.

14. Machens A, Holzhausen HJ, Lautenschlager C, et al. Enhancement of lymph node metastasis and distant metastasis of thyroid carcinoma. Cancer 2003;98:712-9.

15. Adam MA, Thomas S, Hyslop T, et al. Exploring the Relationship Between Patient Age and Cancer-Specific Survival in Papillary Thyroid Cancer: Rethinking Current Staging Systems. J Clin Oncol 2016;34:4415-20.

16. Xiong $\mathrm{Y}$, Zhao Q, Liu C, et al. Prognosis of patients with TX stage differentiated thyroid cancer: propensity scored matching analysis of the SEER database 2004-2013. Am J Transl Res 2018;10:2004-14.

17. Liu Y, Liu Z, Zhao Q, et al. Propensity score matching analysis of the prognosis for the rare insular subtype of thyroid cancer based on SEER database. Oncotarget 2017;8:101623-33.

18. Liu C, Chen T, Zeng W, et al. Reevaluating the prognostic significance of male gender for papillary thyroid carcinoma and microcarcinoma: a SEER database analysis. Sci Rep 2017;7:11412.

19. Goffredo P, Sosa JA, Roman SA. Differentiated thyroid cancer presenting with distant metastases: a population analysis over two decades. World J Surg 2013;37:1599-605.

20. Jeon MJ, Kim WG, Choi YM, et al. Features Predictive of Distant Metastasis in Papillary Thyroid Microcarcinomas. Thyroid 2016;26:161-8. 
21. Sun R, Zhang H, Liu K, et al. Clinicopathologic Predictive Factors of Cervical Lymph Node Metastasis in Differentiated Thyroid Cancer. Acta Otorrinolaringol Esp 2018;69:149-55.

Cite this article as: Liu Z, Chen S, Huang Y, Hu D, Zeng W, Wang M, Zhou W, Chen D, Feng H, Wei W, Zhang C, Zhou L, Guo L. Synergic effects of histology subtype, tumor size, and lymph node metastasis on distant metastasis in differentiated thyroid cancer. Ann Transl Med 2019;7(20):533. doi: 10.21037/ atm.2019.09.137
22. Shi RL, Qu N, Yang SW, et al. Tumor size interpretation for predicting cervical lymph node metastasis using a differentiated thyroid cancer risk model. Onco Targets Ther 2016;9:5015-22. 


\section{Supplementary}

Table S1 Measures for estimation of synergic effect between tumor size (20 mm as cut-off values) and N stage for the risk of distant metastasis in DTC

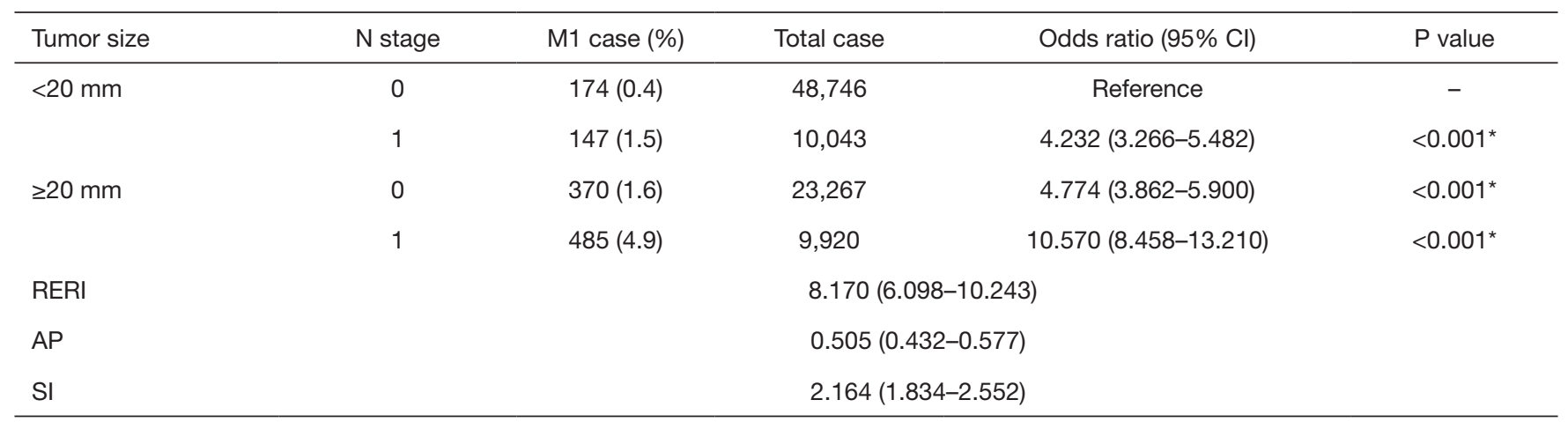

Adjusted for age at diagnosis, sex, race, histology subtype, extrathyroidal extension. *, represent the $\mathrm{P}$ value $<0.05$. DTC, differentiated thyroid cancer; Cl, confidence interval; RERI, relative excess risk; AP, attributable proportion; SI, synergy index.

Table S2 Measures for estimation of synergic effect between tumor size ( $40 \mathrm{~mm}$ as cut-off values) and N stage for the risk of distant metastasis in DTC

\begin{tabular}{|c|c|c|c|c|c|}
\hline Tumor size & $\mathrm{N}$ stage & M1 case $(\%)$ & Total case & Odds ratio (95\% Cl) & $P$ value \\
\hline$<20 \mathrm{~mm}$ & 1 & $353(2.1)$ & 16,910 & 3.301 (2.760-3.949) & $<0.001^{*}$ \\
\hline \multirow[t]{2}{*}{$\geq 20 \mathrm{~mm}$} & 0 & $210(3.0)$ & 7,034 & $4.656(3.830-5.661)$ & $<0.001^{*}$ \\
\hline & 1 & $279(9.1)$ & 3,053 & $10.456(8.548-12.788)$ & $<0.001^{*}$ \\
\hline AP & \multicolumn{5}{|c|}{$0.576(0.507-0.645)$} \\
\hline SI & \multicolumn{5}{|c|}{$2.518(2.111-3.004)$} \\
\hline
\end{tabular}

Adjusted for age at diagnosis, sex, race, histology subtype, extrathyroidal extension. ${ }^{*}$ represent the $\mathrm{P}$ value $<0.05$. DTC, differentiated thyroid cancer; $\mathrm{Cl}$, confidence interval; RERI, relative excess risk; AP, attributable proportion; SI, synergy index.

Table S3 Measures for estimation of synergic effect between histology subtype and tumor size for the risk of distant metastasis in DTC

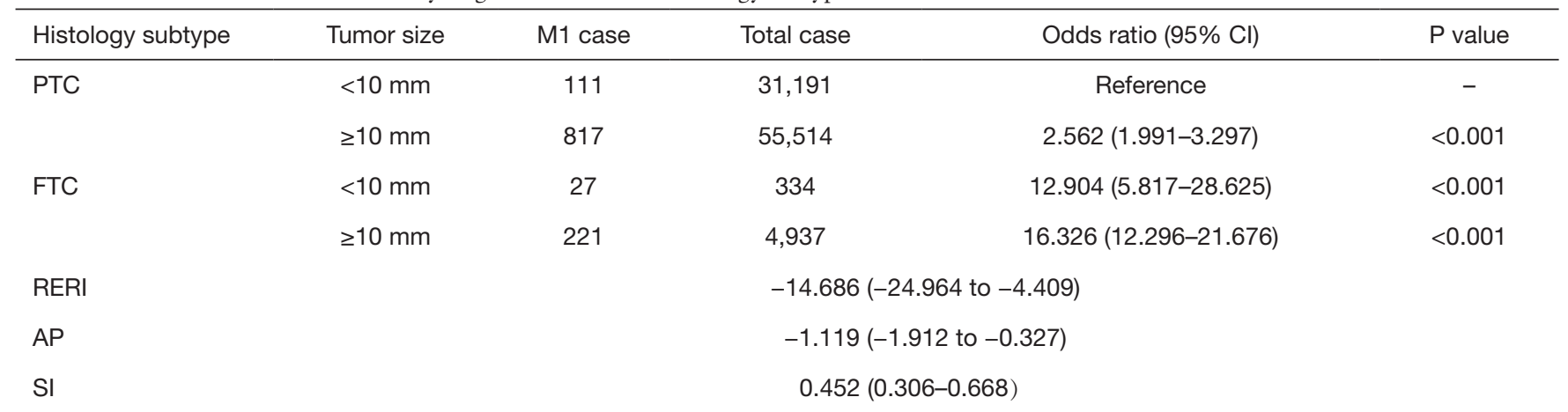

Adjusted for age at diagnosis, sex, race, $\mathrm{N}$ stage, extrathyroidal extension. ${ }^{*}$, represent the $\mathrm{P}$ value $<0.05$. DTC, differentiated thyroid cancer; PTC, papillary thyroid cancer; FTC, follicular thyroid cancer; Cl, confidence interval; RERI, relative excess risk; AP, attributable proportion; SI, synergy index. 
Table S4 The association between two therapies and cancer specific survival of differentiated thyroid cancer

\begin{tabular}{|c|c|c|c|c|c|c|c|c|c|}
\hline & & \multicolumn{4}{|c|}{ Univariate Cox regression analysis } & \multicolumn{4}{|c|}{ Multivariate Cox regression analysis } \\
\hline & & $\mathrm{HR}$ & Lower limit & Upper limit & $P$ value & $\mathrm{HR}$ & Lower lim & pper limit & $P$ value \\
\hline \multirow[t]{2}{*}{ Radiation } & None or refused & Ref. & - & - & - & Ref. & - & - & - \\
\hline & $\begin{array}{l}\text { Radiation beam or radioactive } \\
\text { implants }\end{array}$ & 15.656 & 13.378 & 18.322 & 0.000 & 5.015 & 3.978 & 6.322 & 0.000 \\
\hline \multirow[t]{2}{*}{ Surgery } & Lobectomy & Ref. & - & - & - & Ref. & - & - & - \\
\hline & $\begin{array}{l}\text { Subtotal or near total } \\
\text { thyroidectomy }\end{array}$ & 1.986 & 1.410 & 2.796 & 0.000 & 1.442 & 0.959 & 2.168 & 0.079 \\
\hline
\end{tabular}

Adjusted for age at diagnosis, sex, race, tumor size, lymph node metastasis, multifocality, histology subtype, extrathyroidal extension and surgery (or radiation) in the multivariate Cox regression analysis. $\mathrm{HR}$, hazard ratio; $\mathrm{Cl}$, confidence interval. 